\title{
Bedürfniskarten im Coaching
}

\section{Ein Tool, um im Coaching besser die Bedürfnisse der Klienten besprechbar zu machen}

\author{
Sandra J. Schiemann ${ }^{1}$ (D) · Vicky König ${ }^{1} \cdot$ Maximilian M. A. Brantl $\left.\right|^{1}$ Eva Jonas ${ }^{1}$ (D) \\ Online publiziert: 9. April 2020 \\ (c) Der/die Autor(en) 2020
}

\section{Zusammenfassung}

Bildkarten werden in vielen Coachings eingesetzt, damit die KlientInnen besser ihre Gedanken, Emotionen und/oder Ziele veranschaulichen können. Die Frage ist, ob Bildkarten auch dabei helfen können, damit verbundene grundlegende Bedürfnisse zu erkennen. Daher haben wir in zwei Vorstudien ein Bedürfniskarten-Set entwickelt, das auf den drei psychologischen Grundbedürfnissen nach Autonomie, Kompetenz und sozialer Eingebundenheit (entsprechend der Selbstbestimmungstheorie) basiert. Um zu sehen, wie die Bedürfniskarten auf Coaches wirken, haben wir 332 Coaches befragt, ob bzw. wie sie Bildkarten anwenden sowie wie sie die entwickelten 30 Bedürfniskarten bezüglich der drei Grundbedürfnisse auf einer Adjektivskala einschätzen. Die Ergebnisse zeigten, dass Bildkarten sehr häufig im Coaching verwendet werden und der Einsatz von Bildkarten dabei vorwiegend der Visualisierung und Verankerung von Zielen und Emotionen dient. Darüber hinaus wurden die von uns entwickelten Bedürfniskarten von den Coaches überwiegend den jeweils dazugehörigen Bedürfnissen passend zugeordnet. Eine Zuordnung auf Seiten der Klienten steht noch aus.

Schlüsselwörter Bildkarten · Bedürfniskarten · Coaching $\cdot$ Bedürfnisse $\cdot$ Selbstbestimmungstheorie

\section{Need cards in coaching}

A tool to better discuss the clients' underlying basic psychological needs

\begin{abstract}
Pictures and picture cards are regularly used in coachings to visualize the clients' thoughts, emotions, and/or goals. Thus, the question arises whether picture cards can also help detect the clients' underlying basic needs. To investigate this research question, we developed need cards based on the three basic psychological needs of autonomy, competence, and relatedness (with regard to the Self-Determination Theory). In a survey asking 332 coaches, we explored the coaches' use of picture cards and the fit of the self-developed need cards to a scale based on the three basic psychological needs. The results show that picture cards are frequently used in coaching-mainly to visualize and anchor goals and emotions. The results further reveal that the developed need cards fit to the respective need. Further research with clients as participants is planned.
\end{abstract}

Keywords Pictures $\cdot$ Needs $\cdot$ Coaching $\cdot$ Self-determination theory $\cdot$ Picture cards

Sandra J. Schiemann

sandra.schiemann@sbg.ac.at

Vicky König

vicky.koenig@sbg.ac.at

Maximilian M. A. Brantl

m.brantl@icloud.com
Eva Jonas

eva.jonas@sbg.ac.at

1 Abteilung Sozialpsychologie, Fachbereich Psychologie, Universität Salzburg, Hellbrunner Str. 34, 5020 Salzburg, Österreich 


\section{Theoretischer Hintergrund}

Coaching ist eine Maßnahme zur „Förderung der Reflexionen und Selbstreflexionen sowie Beratung von Personen oder Gruppen zur Verbesserung der Erreichung selbstkongruenter Ziele oder zur bewussten Selbstveränderung und Selbstentwicklung" (Greif 2008, S. 59). Um den Klienten bei der Zielfindung und Zielerreichung zu unterstützen, nimmt der Coach die Rolle eines Prozessbegleiters ein (Rauen 2014). Als Prozess-Experte oder Prozess-Berater unterstützt daher der Coach die Klienten mit ihren selbstgewählten Zielen und in ihrem selbstbestimmten Handeln (Bachkirova und Smith 2015; Schiemann et al. 2018). Dabei gehen Coach und Klient in eine soziale Interaktion, bei der in der Zusammenarbeit zwischen Coach und Klienten gemeinsam ein Ergebnis erarbeitet wird, d.h. Coach und Klient befinden sich in einer Situation der Interdependenz und sind damit in ihrem Erfolg wechselseitig voneinander abhängig (Greif et al. 2012; Jonas et al. 2019; siehe auch Kelley et al. 2003). Um soziale Interaktionen positiv zu gestalten, ist es wichtig, die Bedürfnisse des Gegenübers zu identifizieren und auf diese einzugehen (vgl. Loop2LoopModell; Jonas und Mühlberger 2017). Häufig ist es dabei im Coachingprozess notwendig, die unbewussten Bedürfnisse der Klienten zu aktivieren (Rauen et al. 2011).

\subsection{Bedürfnisse im Coaching}

Grundlegende Bedürfnisse können physiologischer Art sein wie Essen, Trinken oder Schlafen, aber es gibt auch psychologische Grundbedürfnisse wie das Bedürfnis nach Autonomie, Kompetenz und sozialer Eingebundenheit (Selbstbestimmungstheorie; Ryan und Deci 2017). Autonomie ist durch selbstverantwortliches und -bestimmtes Handeln, das mit den Werten, Vorstellungen und Zielen der Person kongruent ist, gekennzeichnet. Kompetenz meint das erfolgreiche Bewältigen schwieriger, herausfordernder Situationen und das Gefühl, selbst zu der Veränderung einer Situation beigetragen zu haben. Soziale Eingebundenheit bedeutet, dass Individuen sich nahe und sicher gebunden fühlen in Bezug auf Personen in ihrer Lebenswelt und der Gesellschaft, sowie dass sie ein klares Bild von ihren Aufgaben und Rollen haben (Gagné und Deci 2005; Ryan und Deci 2017). Diese Bedürfnisse bestimmen das menschliche Handeln, tragen maßgeblich zur Motivation bei und steuern unser Verhalten (Storch und Kuhl 2012). Daher werden Bedürfnisse auch als Energiequellen für das menschliche Verhalten bzw. als Grundlage der menschlichen Motivation verstanden (Deci und Ryan 1993). Jedoch sind Bedürfnisse nicht nur die Initiatoren von motiviertem Verhalten, sondern bestimmen auch Wohlbefinden, Wachstum und $\mathrm{Zu}$ friedenheit (Ryan und Deci 2000a, 2000b). Beispielsweise führt die Befriedigung aller drei Grundbedürfnisse am Ar- beitsplatz zu besseren Arbeitsleistungen, mehr Arbeitsengagement und zu einer positiveren Einstellung (Baard et al. 2004; Deci et al. 2001). Darüber hinaus können Bedürfnisse durch die Ausgestaltung des Beratungsprozesses unterstützt oder vernachlässigt werden (Böhm et al. 2017; Jonas et al. 2019). Dementsprechend ist die Identifikation der Bedürfnisse durch den Klienten (und den Coach) eine wichtige Vorrausetzung für ein erfolgreiches Coaching.

\subsection{Bilder zum Erkennen von Bedürfnissen im Coaching}

Allein der verbale Austausch reicht im Coaching jedoch häufig nicht aus, um einen Zugang zu unbewussten Bedürfnisse zu bekommen (Messerschmidt 2015). Um die Bedürfnisse der Klienten zu erkennen, helfen unter anderem Coachingtools, die die Problem- und Selbstreflexion anregen (Greif 2009; Wechsler 2012). Dabei können Bildkarten im Coaching unterstützen, indem sie innere Gedanken verbildlichen (Larro-Jacob 2007), eigene Stimmungen visualisieren (Harrn und Teusianu 2017; Hofmann, 2019), Ziele darstellen (Gut und Kühne-Eisendle 2014) und eigene Ressourcen aktivieren (Deubner-Böhme und DeppeSchmitz 2018a, 2018b; Krause und Storch 2011). Auch in der Therapie gilt die Arbeit mit Bildern als Möglichkeit, Inneres zu erforschen und somit innere Veränderung zu erzielen (Gärd et al. 2019). Zusammengefasst können bildhafte Methoden also implizite Bedürfnisse anregen, bewusstmachen und in Ziele übersetzen (Kuhl und Strehlau 2011; Rauen et al. 2011; Storch 2011). Messerschmidt (2015, 2018) zeigte die Nützlichkeit von Bildern im Coaching zur besseren ergebnisorientierten Selbstreflexion, um Bedürfnisse zu erkennen und zu bearbeiten: Sie führte Experteninterviews mit Coaches und Klienten zu ihren subjektiven Erfahrungen zu Bildkarten durch und fand heraus, dass die Bildkarten die Perspektive des Klienten erweiterten, zu mehr Klarheit beim Klienten führten, sowie den Zugang zu Emotionen vereinfachten.

Bildkarten-Sets gibt es in der Therapie wie im Coaching viele (z.B. Fräntzel und Johannsen 2019; Nührig 2019; Weidenmann und Weidenmann 2013; Wicher 2019). Die Arbeit mit Bildkarten erfolgt dabei meist intuitiv, das heißt, es wird vor allem ,nach Gefühl“ und nicht nach einem durchdachten Konzept gearbeitet (z.B. Erwachsenenbildung 2014). Es gibt also meist kein wissenschaftliches Fundament, welches das Vorgehen stützt. Die Experteninterviews von Messerschmidt $(2015,2018)$ zeigten, dass von selbst zusammengestellten Postkarten- oder Fotosammlungen bis hin zur ZRM-Bildkartei (Krause und Storch 2011) alles verwendet wird; auch werden die gleichen Bildkarten für unterschiedliche Fragestellungen verwendet, so dass der nachfolgende Dialog meist erst die Bedeutung des Bildes aufklärte. 

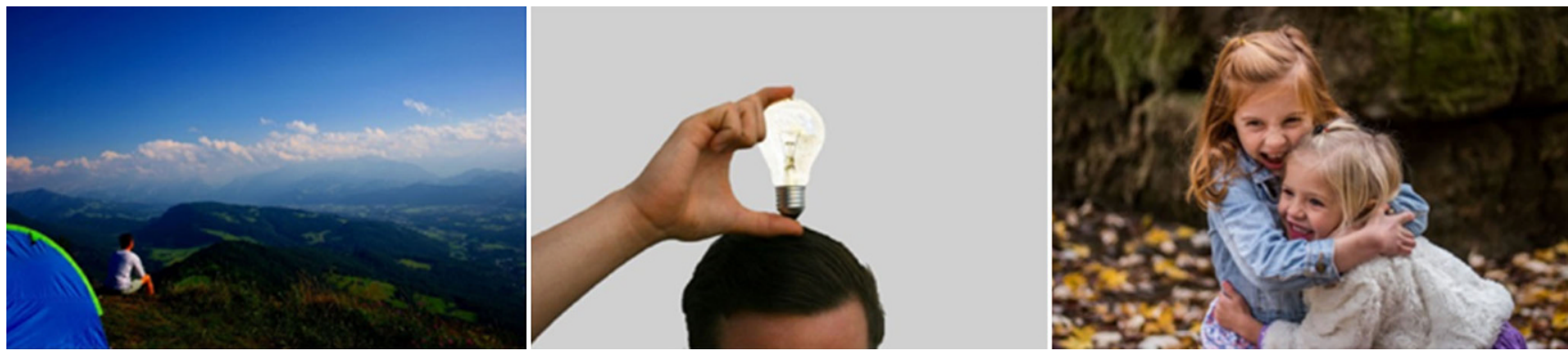

Abb. 1 Beispiel-Bilder für die Bedürfnisse nach Autonomie, Kompetenz und soziale Eingebundenheit (von links nach rechts)

Auch wenn diese Arbeit mit Bildkarten sehr wirksam sein kann, steckt in Bildkarten unseres Erachtens viel mehr Potential: Bilder können symbolisch-nonverbal für die dahinterliegenden Bedürfnisse stehen (Storch 2010; siehe auch Bucci 2008). In anderen Worten kann ein Bild aufzeigen, was eine Person braucht, da ein Bild abstrahiert und damit den Zugang zum Extensionsgedächtnis erleichtert (Kuhl 2001; Storch 2010). Messerschmidt (2015, 2018) schilderte in ihren Ergebnissen, dass die Bilder wie eine Brücke zum inneren Selbst des Klienten fungieren - und damit einen Zugang zu dessen Bedürfnissen und unbewussten Zielen bieten. Können daher Bilder nicht nur den Zugang zu den Bedürfnissen erleichtern, sondern sogar für bestimmte dahinterliegende Bedürfnisse stehen?

\subsection{Die vorliegende Studie: Bedürfniskarten im Coaching}

Um diese Frage $\mathrm{zu}$ untersuchen, haben wir mithilfe von Experteninterviews ein Bilderset erstellt, welches die drei psychologischen Grundbedürfnisse nach Autonomie, Kompetenz und soziale Eingebundenheit umfassen soll. Ähnlich wie beim Operanten Motivtest (OMT; Scheffer et al. 2003) soll ein Bild für ein bestimmtes Bedürfnis stehen, jedoch sollen die Bilder wie im Züricher Ressourcen Modell (Storch 2010) nur positive Bedeutungen haben. Außerdem sollen anders als beim OMT bei unseren Bedürfniskarten keine qualitativen Aussagen zum Bild ausgewertet werden. In zwei Vorstudien wurden dann die Bedürfniskarten per Online-Umfragen getestet und überprüft, inwiefern die Bilder zu den drei Grundbedürfnissen passten ${ }^{1}$. Das fertige Bilderset (30 Bedürfniskarten mit je 10 Karten pro Bedürfnis;

\footnotetext{
1 In der ersten Vorstudie $(N=11)$ wurden 15 Bilder für Autonomie, 12 für Kompetenz und 11 für Soziale Eingebundenheit getestet, inwieweit sie bezüglich der Adjektivskala (siehe Methode dieses Papers) $\mathrm{zu}$ den Bedürfnissen passen. Die Ergebnisse zeigten, dass alle Bilder für Soziale Eingebundenheit auf der Skala soziale Eingebundenheit die höchsten Werte hatten und es keine Überscheidungen mit den Konfidenzintervallen der anderen Skalen gab; von den 15 Bildern für Autonomie waren sieben eindeutig der Skala Autonomie zuzuordnen; bezüglich der 12 Bilder für Kompetenz waren 3 eindeutig zuzuordnen. In einem weiteren Coaching-Experteninterview wurden daher vier weite-
}

siehe Abb. 1) sollte dann in einem ersten systematischen Validierungsschritt von Coaches beurteilt werden. Wir waren demnach in dieser Studie daran interessiert zu erfahren, wie Bildkarten verwendet werden und ob unsere Bedürfniskarten zu den jeweiligen Bedürfnissen passen.

\section{Methode}

\subsection{Stichprobe}

Im Rahmen einer Befragung, die mit Unterstützung von XING Coaches + Trainer durchgeführt wurde, konnten die im Folgenden analysierten Daten erhoben werden. Hierbei wurden 332 Coaches (186 Frauen, 146 Männer) befragt, die auf der Online-Plattform XING Coaches + Trainer ein aktives Profil angelegt hatten. Die Coaches waren zwischen 23 und 77 Jahre alt $(M=51,70, S D=9,36)$, hatten bereits eins bis 5000 Klienten $(M=320,70, S D=500,00)$ und hatten größtenteils eine zertifizierte Coachingausbildung (64\%).

\subsection{Ablauf}

Die Coaches wurden mit Hilfe einer Benachrichtigung zur Befragung eingeladen und nach Bestätigung des Links auf das online Befragungstool (LimeSurvey 2.67.3) weitergeleitet. Nach einer Information zur Studie und darüber, dass alle Angaben freiwillig, anonym und vertraulich sind sowie die Befragung jederzeit abgebrochen werden kann, erhielten die Coaches Fragen zu ihnen als Person, zu ihrem Einsatz verschiedener Coachingtools inklusive von Bildkarten, sowie dem von uns erstellten Bedürfniskarten-Set am Ende mit einer Adjektivskala pro Bild. Zum Abschluss wurde ihnen für die freiwillige Teilnahme bedankt und angebo-

re Bilder für Autonomie und 16 weitere Bilder für Kompetenz ergänzt. Eine zweite Vorstudie $(N=10)$ zeigte, dass nun jeweils 10 Bilder für Autonomie, Kompetenz und soziale Eingebundenheit gut abgrenzbar funktionierten. Die Ergebnisse dieser zweiten Vorstudie sowie weitere Experteninterviews bildeten die Basis für das in dieser Studie evaluierte Bedürfniskarten-Set. 
Tab. 1 Adjektiv-Aussage-Skala zu den drei Bedürfnissen in der angegebenen Reihenfolge

Bitte bewerten Sie im Folgenden, inwiefern die genannten Adjektive auf das Bild zutreffen:

\begin{tabular}{lll}
\hline 1. & Positiv & (Nicht Teil der Skala) \\
2. & Autonom & Autonomie \\
3. & Qualifiziert & Kompetenz \\
4. & Im Einklang mit mir selbst & Autonomie \\
5. & Gemeinsam mit anderen & Soziale Eingebundenheit \\
6. & Zusammen mit anderen & Soziale Eingebundenheit \\
7. & Selbstbestimmt & Autonomie \\
8. & Fähig & Kompetenz \\
9. & Verbunden & Soziale Eingebundenheit \\
10. & Kompetent & Kompetenz \\
\hline
\end{tabular}

Zur Erleichterung für die Bewertung der 30 Bilder wurden diese Adjektive nur vorab per Zufall randomisiert aber dann in genau der randomisierten Reihenfolge gleich behalten

Tab. 2 Rotierte Komponentenmatrix (Faktorenanalyse)

\begin{tabular}{llll}
\hline & Komponenten & & 3 (soz. Eingebundenheit) \\
\cline { 2 - 4 } & 1 (Autonomie) & 2 (Kompetenz) & 0,273 \\
\hline Autonom & 0,885 & 0,318 & 0,312 \\
Im Einklang mit mir selbst & 0,860 & 0,372 & 0,345 \\
Selbstbestimmt & 0,789 & 0,431 & 0,391 \\
Qualifiziert & 0,537 & 0,735 & 0,422 \\
Fähig & 0,500 & 0,743 & 0,416 \\
Kompetent & 0,475 & 0,748 & 0,897 \\
Zusammen mit anderen & 0,266 & 0,290 & 0,914 \\
Gemeinsam mit anderen & 0,273 & 0,246 & 0,788 \\
Verbunden & 0,315 & 0,351 & \\
\hline
\end{tabular}

Die drei Faktoren erklären zusammen $95 \%$ der Varianz

Tab. 3 Bilder und Bedürfnisskala

\begin{tabular}{|c|c|c|c|c|c|c|}
\hline \multirow{2}{*}{$\frac{\text { Bilder }}{\text { Autonomie-Bilder }}$} & \multicolumn{2}{|c|}{ Autonomie: $M(S D)$} & \multicolumn{2}{|c|}{ Kompetenz: $M(S D)$} & \multicolumn{2}{|c|}{ Soz.E.: $M(S D)$} \\
\hline & 4,25 & $(1,18)$ & 3,26 & $(1,43)$ & 1,46 & $(0,96)$ \\
\hline Kompetenz-Bilder & 3,26 & $(1,36)$ & 3,75 & $(1,43)$ & 1,44 & $(1,09)$ \\
\hline Soz.E.-Bilder & 2,68 & $(1,36)$ & 2,51 & $(1,48)$ & 4,82 & $(1,09)$ \\
\hline
\end{tabular}

ten, dass sie die Ergebnisse der Studie per Mail zugesendet bekommen.

\subsection{Messmethoden}

Verwendung von Coachingtools Nach den demografischen Daten fragten wir die Coaches, wie häufig sie Übungen, Tests, Bildkarten und Beratungsanteile verwenden mit Antwortmöglichkeiten auf einer Likertskala von 1 (nie) über 4 (2-3mal pro Coaching) bis 7 (mehrmals pro Sitzung).

Bedürfniskarten mit Adjektivskala pro Bild Um die drei Grundbedürfnisse der Selbstbestimmungstheorie von Deci und Ryan (2000, 2002) zu erfassen, wurde die AdjektivAussage-Skala (Brantl et al. 2017; siehe Tab. 1) verwendet. Darüber hinaus wurde auf der gleichen Skala gefragt, wie „positiv“ das Bild wahrgenommen wird. Dabei sollten die KlientInnen auf einer Likertskala von 1 (trifft überhaupt nicht $z u$ ) bis 6 (trifft voll und ganz zu) angeben, inwiefern die genannten Adjektive auf das Bild zutrafen.

Die Reliabilitäten der Bedürfnis-Subskalen waren über alle Bilder hinweg sehr gut (Autonomie-Skala: $\alpha=0,97$, Kompetenz-Skala: $\alpha=0,99$, Soz. Eingebundenheit-Skala: $\alpha=0,95$ ). Auch die Faktorenanalyse mit 3-Faktorenstruktur und Varimax-Rotation der gemittelten Adjektivskala ergab über alle Bilder hinweg ein sehr passendes Bild (siehe Tab. 2).

\subsection{Statistische Auswertung}

Für die Datenanalyse wurde das statistische Berechnungsprogramm IBM Statistics SPSS.24 verwendet. Neben de- 
skriptiven Datenanalysen wurde für das BedürfniskartenSet eine ANOVA mit Messwiederholung mit Kontrasten sowie eine Faktorenanalyse mit vorgegebener Drei-FaktorenStruktur berechnet.

\section{Ergebnisse}

\subsection{Verwendung von Bildkarten im Vergleich zu anderen Coachingtools}

Wir konnten zeigen, dass Bildkarten im Coaching durchschnittlich bei jedem Coachingprozess verwendet werden $(M=3,03, S D=1,67)$ und dabei öfter als Tests $(M=1,23$, $S D=1,28)$ oder Beratungsanteile $(M=1,93, S D=1,80)$, sowie fast so häufig wie insgesamt alle anderen Übungen $(M=3,32, S D=1,61)^{2}$. Coaches, die Bildkarten einsetzten ( $n=327)$, kommentierten häufig, dass sie Bilder als Gesprächsbasis zur Visualisierung und Verankerung von Emotionen und Zielen verwenden. Zudem haben wir gefragt, auf welcher Erfahrungs- oder theoretischen Basis sie Bildkarten im Coaching verwenden. Hier gaben die meisten an, dass sie auf Basis eigener Erfahrungen, einer ZRM-Ausbildung, und/oder der eigenen Coachingausbildung Bildkarten verwenden.

\subsection{Testung der Bedürfniskarten}

Von den teilnehmenden Coaches haben 79 Personen die Karten anhand der Adjektive vollständig bewertet und 59 Personen zu einem ausreichend großen Anteil (297-299 von insg. 300 Adjektiven markiert), so dass insgesamt die Antworten von 139 Personen ausgewertet werden können. Die Ergebnisse zeigen vorab, dass alle Bilder überwiegend positiv wahrgenommen wurden $(M=3,81, S D=1,13)$. Bei der Berechnung einer ANOVA mit Messwiederholung inklusive Kontraste pro Bild, zeigte sich, dass die Bilder das dazugehörige Bedürfnis ansprechen (siehe Tab. 2). In anderen Worten hatte bei den Autonomie-Bildern die Skala Autonomie über alle Bilder hinweg signifikant höhere Mittelwerte als die Skalen Kompetenz, $p<0,001$, und soziale Eingebundenheit, $p<0,001, F(2,276)=424,67$, $\eta^{2}=0,76$. Bei den Kompetenz-Bildern zeigte die Skala Kompetenz signifikant höhere Mittelwerte als die Skala Soziale Eingebundenheit, $p<0,001$, und bei sechs der zehn Bilder auch höhere Mittelwerte als die Skala Autonomie, $p<0,001, F(2,276)=346,41, \eta^{2}=0,72$. Bei den

\footnotetext{
${ }^{2}$ Coaches, die Übungen einsetzten $(n=327)$ kannten diese meist aus einer Coaching- $(80 \%)$ oder Trainings-/Beratungsausbildung (64\%), sowie hatten die Übung selbst kreiert $(71,6 \%)$. In der qualitativen Analyse der Frage zur Lieblingsübung zeigte sich, dass Kreativitätsübungen, systemische Übungen und Achtsamkeitsübungen besonders beliebt waren.
}

Soziale-Eingebundenheit-Bildern weist die Skala Soziale Eingebundenheit signifikant höhere Mittelwerte als die Skalen Kompetenz, $p<0,001$, und Autonomie, $p<0,001$, auf, $F(2,276)=348,84, \eta^{2}=0,72$ (siehe Tab. 3).

\section{Diskussion}

Im Coaching stehen die Bedürfnisse des Klienten im Mittelpunkt, so dass es wichtig ist, dass der Coach die Bedürfnisse der Klienten identifizieren und auf diese eingehen kann (Jonas et al. 2019). Hierfür können unter anderem Coachingtools eingesetzt werden, die die Problem- und Selbstreflexion der Klienten anregen (Greif 2009; Wechsler 2012). Allerdings werden in der Praxis nur selten wissenschaftlich fundierte Methoden verwendet, so dass es wichtig ist, Coachingtools und deren Wirkung zu erforschen (ICF 2016). Ein erstes Ergebnis der Studie ist, dass die befragten Coaches angaben, häufig mit Bildern zu arbeiten. Dieses Ergebnis unterstreicht, wie wichtig Bilder im Coaching sind und wie leicht es wäre, an dieser Methode anzuknüpfen. Vereinzelt gibt es bereits Hinweise, dass durch den Einsatz von Bildern der Zugang zu den eigenen Bedürfnissen erleichtert wird, indem diese symbolisch-nonverbal die dahinterliegenden Bedürfnisse spiegeln (Messerschmidt 2015, 2018; Storch 2010). Die Idee dieser Studie war daher, Bilder für Bedürfnisse zu finden, um Coaches darin zu unterstützen Bedürfnisse zielgerichteter zu erkennen, zu benennen und zu unterstützen.

Aus diesem Grund entwickelten wir Bedürfniskarten, die auf den drei psychologischen Grundbedürfnissen der Selbstbestimmungstheorie (Deci und Ryan 2015) nach Autonomie, Kompetenz und sozialer Eingebundenheit basieren, um dem Coach das Identifizieren und Erkennen der Bedürfnisse zu erleichtern. Werden diese Bedürfnisse erfüllt, so führt dies zu mehr Zufriedenheit, Gesundheit, Wachstum und Wohlbefinden (Deci und Ryan 2015; Deci et al. 2001; Milyavska und Koestner 2010; Ryan et al. 2008). Das Bedürfniskarten-Set bestand aus 10 Bildern für Autonomie, 10 für Kompetenz und 10 für soziale Eingebundenheit. Die Ergebnisse bestärken unsere Vermutung, dass jede Bildkarte einem der drei Grundbedürfnisse der STD von Deci und Ryan (2000, 2002) zuzuordnen ist. In anderen Worten können also Bilder auch ohne Dialog mit dem Klienten bereits eine Aussage über dahinterliegende Bedürfnisse geben.

\subsection{Limitationen und geplante Validierungsschritte}

Bisher wurden die Bedürfniskarten nur anhand der Befragung von Coaches überprüft. In einem nächsten Schritt ist es daher wichtig, die Bedürfniskarten auch in der Verwendung mit echten Klienten zu testen, um zu sehen, ob die Bedürfniskarten auch beim Klienten das jeweilige Bedürfnis 
ansprechen. Aktuell werden daher die von uns entwickelten Bedürfniskarten in Coachings bei Klienten zur Zielvisualisierung eingesetzt und mit Fragebögen zu den eigenen Bedürfnissen begleitet. In diesem Schritt ist es wichtig zu sehen, ob auch hier nur die sechs der zehn Kompetenzbilder höhere Mittelwerte haben als die Autonomiebilder. Erst nach diesen wichtigen Validierungsschritten können die Bedürfniskarten inklusive Anleitungen auf Basis der CoachErfahrungen angeboten werden.

Es muss jedoch klar gesagt werden, dass die Bedürfniskarten keine definitiven Aussagen treffen können und auch nicht sollen, sondern nur als Gesprächsbasis und zur Hypothesengenerierung unterstützen sollen. Sie sind also kein Test und erfüllen daher auch keine konvergente und diskriminante Validitätskriterien (siehe Bühner 2010). Hier unterscheiden sich die Bedürfniskarten beispielsweise von anderen Testverfahren, wie dem OMT (Scheffer et al. 2003). Bilder können mit einer ganz individuellen Erinnerung oder Idee verknüpft sein und daher ist das nachfolgende Gespräch mit dem Klienten besonders wichtig. Die Bilder helfen nur dabei, das nachfolgende Gespräch mit bedürfnisachtsamen Kommunikationstechniken zu gestalten: So kann die Auswahl eines Autonomie-Bildes dazu führen, selbst- versus fremdbestimmt abzuklären oder besonders mit autonomieunterstützenden Techniken zu arbeiten.

\subsection{Theoretische Implikationen}

Zukünftige Forschung sollte untersuchen, ob die Wahl einer Bedürfniskarte als Handlung selbst reicht, um eine situative Bedürfnisbefriedigung zu erreichen. Beispielsweise werden Bilder im Rahmen des Zürcher-Ressourcen-Models (ZRM; Krause und Storch 2011) als emotionale Anker für das angestrebte Ziel des Klienten mithilfe von Verbalisierungsmethoden ,übersetzt“", was eine situative Bedürfnisbefriedigung erhöhen könnte, da das nonverbale Empfinden des Klienten eine verbal-kognitive Bedeutung erhält, die sich für den Klienten kongruent mit dem eigenen Selbst anfühlt. Eine weitere Implikation ist der Einsatz von Bedürfniskarten in anderen Beratungskontexten wie Training oder Mentoring. Hier könnte auch evaluiert werden, ob spezifische Bedürfniskarten sich für thematisch passende Trainingsinhalte besser eignen könnten; es wäre beispielsweise denkbar, dass Klienten in einem Teambuilding-Training eher Soziale Eingebundenheit Bedürfniskarten wählen, während Klienten in einem Stärkentraining eher Kompetenz Bedürfniskarten bevorzugen und ob eine Passung zwischen Bildkarte und Anliegen zu einer höheren Zielerreichung oder Zufriedenheit mit dem Training führt.

\subsection{Praktische Implikationen}

Damit Coaches die Bedürfniskarten effektiv verwenden können, stellt sich nun die Frage nach Einsatzmöglichkeiten für das Bedürfniskarten-Set. Weidenmann und Weidenmann (2013) sehen vielfältige Möglichkeiten, Bildkarten im Coaching zu verwenden - beispielweise zur Zielklärung, zur Selbst- und Beziehungsreflexion, für persönliches Feedback, für Kreativität und Imagination, für Momentaufnahmen. Für die Bedürfniskarten sehen die Autoren vor allem die Möglichkeit, diese in Bezug auf Ziele zu verwenden - zum einen zu Beginn des Coachings bei der Zielklärung und zum anderen in weiteren Coachingphasen bei Zielkonflikten.

Bedürfniskarten zur Zielvisualisierung zu Beginn des Coachings In der Startphase ist es wichtig, ein klares, messbares und auch für den Klienten wichtiges Ziel zu erarbeiten (Lippmann 2009). Ziele sind dabei bewusst kommunizierte Motivationen, die auf dahinterliegenden Bedürfnissen basieren (Storch und Kuhl 2012). Das Wählen einer für das Ziel passenden Bedürfniskarte könnte helfen, diese hinter dem Ziel liegenden Bedürfnisse zu erkennen, abzuklären und besprechbar zu machen. Die Bild-Brücke zu den Bedürfnissen kann dem Coach dabei helfen, das Ziel und mögliche Zielambivalenzen frühzeitig $\mathrm{zu}$ erkennen und besser besprechbar zu machen. Darüber hinaus könnte der Coach das Coaching angepasst an die Bedürfnisse des Klienten gestalten, indem er beispielsweise Trainingssequenzen einbaut, um das Kompetenzbedürfnis zu befriedigen, oder Empathie zeigt, um das Autonomiebedürfnis zu unterstützen (Schiemann et al. 2018). Die Verwendung der Bedürfniskarten zur Zielvisualisierung wird derzeit getestet und erste Erfahrungsberichte von Coaches zeigen, dass es den Klienten sehr leicht fällt, eine Karte für ihr Ziel auszuwählen.

Bedürfniskarten bei Zielkonflikten in weiteren Coachingphasen Auch in weiteren Coachingphasen können die Bedürfniskarten Coach und Klient darin unterstützen, die Bedürfnisse der Klienten genauer zu erforschen und den Coaching Prozess dadurch zu verbessern. Beispielsweise bei späteren Zielkonflikten ist es häufig nötig, auf dahinterliegende Bedürfnisse genauer einzugehen und diese gemeinsam mit dem Klienten zu erforschen. Wird hier verpasst, die hinter Konflikten zugrundeliegenden Bedürfnisse zu erkennen, könnte der Prozess aufgrund von Widerständen ins Stocken geraten (Storch und Kuhl 2012). Hier könnten beispielsweise mit Hilfe der Bedürfniskarten Hypothesen generiert werden, die den Konflikt besprechbar und möglicherweise integrierbar machen. Daher ist es wichtig als Coach hier ein Tool zu haben, das wie ein Kompass in eine mögliche Richtung für die Weiterarbeit 
hinweist. Erste Erfahrungen aus der Coachingpraxis deuten darauf hin, dass Klienten mit Zielkonflikten oft zwei verschiedene Bedürfniskarten wählen anstatt nur eine Karte auszusuchen, was den Coaches dabei hilft, das Ziel in sich entgegenstehende Unterziele herunter zu brechen.

Danksagung Danke an XING Coaches + Trainer für die Weiterleitung unseres Online-Links an ihren Coachingpool.

\section{Einhaltung ethischer Richtlinien}

Interessenkonflikt S.J. Schiemann, V. König, M.M.A. Brantl und E. Jonas geben an, dass kein Interessenkonflikt besteht.

Ethische Standards Diese Forschung wurde vom Ethikkommitee bewilligt und alle TeilnehmerInnen der Studie stimmten zudem einem Informed Consent zu.

Open Access Dieser Artikel wird unter der Creative Commons Namensnennung 4.0 International Lizenz veröffentlicht, welche die Nutzung, Vervielfältigung, Bearbeitung, Verbreitung und Wiedergabe in jeglichem Medium und Format erlaubt, sofern Sie den/die ursprünglichen Autor(en) und die Quelle ordnungsgemäß nennen, einen Link zur Creative Commons Lizenz beifügen und angeben, ob Änderungen vorgenommen wurden.

Die in diesem Artikel enthaltenen Bilder und sonstiges Drittmaterial unterliegen ebenfalls der genannten Creative Commons Lizenz, sofern sich aus der Abbildungslegende nichts anderes ergibt. Sofern das betreffende Material nicht unter der genannten Creative Commons Lizenz steht und die betreffende Handlung nicht nach gesetzlichen Vorschriften erlaubt ist, ist für die oben aufgeführten Weiterverwendungen des Materials die Einwilligung des jeweiligen Rechteinhabers einzuholen.

Weitere Details zur Lizenz entnehmen Sie bitte der Lizenzinformation auf http://creativecommons.org/licenses/by/4.0/deed.de.

\section{Literatur}

Baard, P.P., Deci, E.L., \& Ryan, R.M. (2004). Intrinsic need satisfaction: A motivational basis of performance and well-being in two work settings. Journal of Applied Social Psychology, 34(10), 2045-2068.

Bachkirova, T., \& Smith, C.L. (2015). From competencies to capabilities in the assessment and accreditation of coaches. International Journal of Evidence Based Coaching and Mentoring, 13(2), 123-140.

Böhm, A.M., Mühlberger, C., \& Jonas, E. (2017). Wachstums- und Sicherheitsorientierung im Coaching. In S. Greif, H. Möller \& W. Scholl (Hrsg.), Handbuch Schlüsselkonzepte im Coaching. Springer Reference Psychologie. Berlin, Heidelberg: Springer.

Brantl, M. M. A., Jonas, E., Mühlberger, C. \& Schiemann, S. (2017). Adjektiv-Aussage-Skala. Unveröffentlichter Fragebogen, Universität Salzburg.

Bucci, W. (2008). The referential process, consciusness, and the sense of self. Psychoanalytic Inquiry, 22(5), 766-793.

Bühner, M. (2010). Einführung in die Test- und Fragebogenkonstruktion (3. Aufl.). München: Pearson.

Deci, E.L., \& Ryan, R. M. (1993). Die Selbstbestimmungstheorie der Motivation und ihre Bedeutung für die Pädagogik. Zeitschrift für Pädagogik, 39(2), 223-238.

Deci, E. L., \& Ryan, R. M. (2000). The "what" and "why" of goal pursuits: Human needs and the self-determination of behavior. Psychological Inquiry, 11(4), 227-268.
Deci, E. L., \& Ryan, R. M. (2002). Handbook of self-determination research. New York: University of Rochester Press.

Deci, E.L., \& Ryan, R.M. (2015). Self-determination theory. In J. Wright (Hrsg.), International encyclopedia of the social \& behavioral sciences (S. 486-491). Amsterdam: Elsevier.

Deci, E. L., Ryan, R. M., Gagné, M., Leone, D. R., Usunov, J., \& Kornazheva, B.P. (2001). Need statisfaction, mtivation, and wellbeing in the work organizations of a former Eastern Bloc country. Personality and Social Psychology Bulletin, 27, 930-942.

Deubner-Böhme, M., \& Deppe-Schmitz, U. (2018a). 100 Karten für das Coaching mit Ressourcenaktivierung. Göttingen: Hogrefe.

Deubner-Böhme, M., \& Deppe-Schmitz, U. (2018b). Coaching mit Ressourcenaktivierung. Ein Leitfaden für Coaches, Berater und Trainer. Göttingen: Hogrefe.

Erwachsenenbildung (2014). Bildkarten: Die Wirkung von Bildern in der Lehre nutzen. https://erwachsenenbildung.at/aktuell/nachrich ten_details.php?nid=7429. Zugegriffen: 1 . April 2020

Fräntzel, E., \& Johannsen, D. (2019). 80 Bildkarten für Coaching, Supervision, Training und Psychotherapie. Göttingen: Hogrefe.

Gagné, M., \& Deci, E. L. (2005). Self-determination theory and work motivation. Journal of Organizational Behavior, 26(4), 331-362.

Gärd, H., Roxberg, A., Larsson, I., \& Lundqvist-Persson, C. (2019). Expressions of vitality affects and basic affects during art therapy and their meaning for inner change. International Journal of Art Therapy, 24(1), 30-39.

Greif, S. (2008). Coaching und ergebnisorientierte Selbstreflexion. Theorie, Forschung und Praxis des Einzel- und Gruppencoachings. Göttingen: Hogrefe.

Greif, S. (2009). Grundlagentheorien und praktische Beobachtungen zum Coachingprozess. In B. Birgmeier (Hrsg.), Coachingwissen. Denn sie wissen nicht, was sie tun? (S. 129-144). Wiesbaden: VS.

Greif, S., Schmidt, F., \& Thamm, A. (2012). Warum und wodurch Coaching wirkt - Ein Überblick zum Stand der Theorieentwicklung und Forschung über Wirkfaktoren. Organisationsberatung - Supervision - Coaching, 19(4), 375-390.

Gut, J., \& Kühne-Eisendle, M. (2014). Bildbar. 100 Methoden zum Arbeiten mit Bildern und Fotos im Coaching, Training, in der Ausund Weiterbildung, Therapie und Supervision. Bonn: ManagerSeminare.

Harrn, A., \& Teusianu, M. (2017). Mood Cards: Deute deine Stimmung und Gefühle - für Klarheit, Selbstvertrauen und Gesundheit. München: Irisiana.

Hofmann, T. (2019). SinnBilder - Bildkarten für Psychotherapie, Pädagogik und Coaching. Höchberg: ZKS Verlag.

International Coach Federation (2016). Interview mit Jürgen Bache, 1. Vorsitzender ICF-D. Verfügbar unter http://www.coachfederation. de/files/2016_09_training-aktuell_icf_gcs_2016.pdf

Jonas, E., \& Mühlberger, C. (2017). Editorial: Social cognition, motivation, and interaction: How do people respond to threats in social interactions? Frontiers in Psychology, 8, 1577-1580.

Jonas, E., Mühlberger, C., Böhm, A., \& Esser, V. (2019). Motivkongruenz in sozialen Austausch- und Interdependenzprozessen im Karrieremanagement: Training, Coaching, Mentoring und Supervision in einem sozialpsychologischen Vergleich. In S. Kauffeld \& D. Spurk (Hrsg.), Handbuch Karriere und Laufbahnmanagement (S. 811-845). Berlin: Springer.

Kelley, H.H., Holmes, J. G., Kerr, N.L., Reis, H.T., Rusbult, C.E., \& van Lange, P. A. M. (2003). An atlas of interpersonal situations. Cambridge: Cambridge University Press.

Krause, F., \& Storch, M. (2011). Ressourcen aktivieren mit dem Unbewussten: Manual und ZRM-Bildkartei. Bern: Huber.

Kuhl, J. (2001). Motivation und Persönlichkeit: Interaktionen psychischer Systeme. Göttingen: Hogrefe.

Kuhl, J., \& Strehlau, A. (2011). Handlungspsychologische Grundlagen des Coaching. In B. Birgmeier (Hrsg.), Coachingwissen - Denn sie wissen nicht, was sie tun? (2. Aufl. S. 173-184). Wiesbaden: VS. 
Larro-Jacob, A. (2007). Imaginative Techniken im Coaching. Organisationsberatung, Supervision, Coaching, 14(1), 62-71.

Lippmann, E. (2009). Grundlagen. In E. Lippmann (Hrsg.), Coaching (S. 11-46). Berlin: Springer. https://doi.org/10.1007/978-3-54088952-6.

Messerschmidt, J. (2015). Das Selbst im Bild - Eine empirische Studie zum Einsatz, von Bildmaterialien zur Förderung von Selbstreflexions- und Selbstveränderungsprozessen im Einzelcoaching. Frankfurt am Main: Peter Lang.

Messerschmidt, J. (2018). Das Selbst im Bild: Mit Bildern Selbstreflexion und Veränderung im Coaching wirksam unterstützen. In R. Wegener, et al. (Hrsg.), Wirkung im Coaching (S. 74-84). Göttingen: Vandenhoeck \& Ruprecht. https://doi.org/10.13109/ 9783666402975.

Milyavska, M., \& Koestner, R. (2010). Psychological needs, motivation, and well-being: a test of self-determination theory across multiple domains. Personality and Individual Differences, 50(3), 387-339. https://doi.org/10.1016/j.paid.2010.10.029.

Nührig, A. (2019). Bildkarten für Therapie- und Beratungskontexte. Lernen und Lernstörungen, 8(2), 123-124. https://doi.org/10. 1024/2235-0977/a000267.

Rauen, C. (2014). Coaching (2. Aufl.). Göttingen: Hogrefe.

Rauen, C., Strehlau, A., \& Ubben, M. (2011). Eine integrative Theorie über die grundlegenden Wirkzusammenhänge im Coaching. In B. Birgmeier (Hrsg.), Coachingwissen - Denn sie wissen nicht, was sie tun? (2. Aufl. S. 173-184). Wiesbaden: VS.

Ryan, R. M., \& Deci, E. L. (2000a). Self-determination theory and the facilitation of intrinsic motivation, social development, and wellbeing. American Psychologist, 55(1), 68-78.

Ryan, R.M., \& Deci, E.L. (2000b). The darker and brighter sides of human existence: Basic needs as a unifying concept. Psychological Inquiry, 11(4), 319-338.

Ryan, R.M., \& Deci, E. L. (2017). Self-determination theory: basic psychological needs in motivation, development, and wellness. New York: Guilford.
Ryan, R.M., Patrick, H., Deci, E.L., \& Williams, G.C. (2008). Facilitating health behaviour change and its maintenance: Interventions based on Self-Determination Theory. European Psychologist, 10(1), 2-5.

Scheffer, D., Kuhl, J., \& Eichstaedt, J. (2003). Der Operante MotiveTest (MT): Inhaltsklassen Auswertung, psychometrische Kennwerte und Validierung. In J. Stiensmeier-Pelster \& F. Rheinberg (Hrsg.), Diagnostik von Motivation und Selbstkonzept (S. 151-167). Göttingen: Hogrefe.

Schiemann, S. J., Mühlberger, C., \& Jonas, E. (2018). Striving for autonomy: the importance of the autonomy need and its support within coaching. Journal of Evidence-Based Coaching and Mentoring, Special Issue, 12(Special Issue), 98-110.

Storch, M. (2010). Motto-Ziele und Motivation: Wie Ziele mit Kraft, Lust und hoher Wirksamkeit entwickelt werden. Müllheim: Auditorium Netzwerk.

Storch, M. (2011). Embodiment im Zürcher Ressourcenmodell (ZRM). In M. Storch, B. Cantieni, G. Hüther \& W. Tschacher (Hrsg.), Embodiment: Die Wechselwirkung von Körper und Psyche verstehen und nutzen (2. Aufl. S. 127-142). Bern: Huber.

Storch, M., \& Kuhl, J. (2012). Die Kraft aus dem Selbst. Sieben PsychoGyms für jeden Tag. Bern: Huber.

Wechsler, T. (2012). Das coachingtool-spezifische Wirkfaktorenmodell. Organisationsberatung, Supervision, Coaching, 19(4), 405-423.

Weidenmann, S., \& Weidenmann, B. (2013). Bildkarten für Coaching und Beratung. Weinheim: Beltz.

Wicher, M. (2019). (Intra)intermediärobjekte und Symbole in der Psychodrama-Psychotherapie: Unterschiedliches und Gemeinsames. Zeitschrift für Psychodrama und Soziometrie, 18, 211-222.

Publisher's Note Springer Nature remains neutral with regard to jurisdictional claims in published maps and institutional affiliations. 